\title{
Cardiac surgery during pregnancy: Pulsatile or nonpulsatile perfusion?
}

\author{
Marjan Jahangiri, FRCS, ${ }^{a}$ James Clark, FRCA, ${ }^{\text {b }}$ Fredrico Prefumo, MD, ${ }^{c}$ Charles Pumphrey, FRCP, ${ }^{\mathrm{d}}$ and \\ David Ward, FRCP, ${ }^{d}$ London, United Kingdom
}

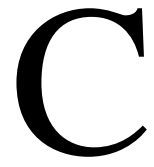

ardiopulmonary bypass (CPB) during pregnancy is associated with a high fetal and maternal mortality. Contributing factors are timing of the operation, emergency situations, and placental vasoconstriction. Experimental evidence suggests that pulsatile perfusion might prevent placental vasoconstriction. We report on 4 patients who underwent cardiac surgery during pregnancy with pulsatile perfusion and detailed fetal monitoring.

\section{Clinical Summaries}

PATIENT 1. A 31-year-old woman at 11 weeks' gestation presented with palpitation. Transthoracic echocardiography showed severe aortic regurgitation and dilated aortic root $(6.9 \mathrm{~cm})$. She underwent aortic root replacement at 12 weeks' gestation with a $27-\mathrm{mm}$ porcine valve and a $30-\mathrm{mm}$ Gelseal graft (Vascutek, Renfrenshire, Scotland). CPB was weaned with no support. The patient was extubated 3 hours after the operation and was discharged at 8 days. Histologic examination confirmed cystic medial necrosis.

PATIENT 2. A 26-year-old woman at 19 weeks' gestation presented with acute shortness of breath. She had undergone open aortic valvotomy at age 15 years for congenital aortic stenosis. Transthoracic echocardiography showed a gradient of $120 \mathrm{~mm} \mathrm{Hg}$ across the valve. She underwent redo aortic valve replacement with a 23-mm porcine valve. She was weaned off CPB with no support, was extubated 4 hours after the operation, and was discharged at 10 days.

PATIENT 3. A 28-year-old woman at 24 weeks' gestation presented with chest pain. Transesophageal echocardiography showed a dilated ascending aorta $(7.2 \mathrm{~cm})$ with aortic regurgitation. She underwent aortic root replacement with a $25-\mathrm{mm}$ porcine valve and a $28-\mathrm{mm}$ Gelseal graft. The operation was uneventful. She was extubated 4 hours after the operation and was discharged at 7 days. Histologic examination confirmed cystic medial necrosis.

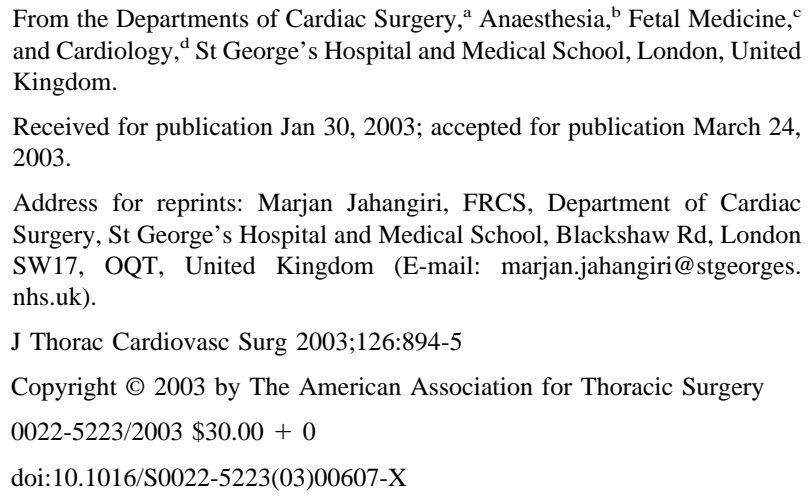

PATIENT 4. A 38-year-old woman at 15 weeks' gestation presented with severe shortness of breath. Transesophageal echocardiography showed severe mitral stenosis, aortic stenosis with a gradient of $55 \mathrm{~mm} \mathrm{Hg}$, and an annulus of $14.5 \mathrm{~mm}$. She underwent aortic and mitral valve replacements with 21 - and $25-\mathrm{mm}$ porcine valves, respectively, and enlargement of the aortic root. She was weaned off $\mathrm{CPB}$ with no support, was extubated 4 hours after the operation, and was discharged home at 6 days.

Management of CPB. All operations were performed at $35^{\circ} \mathrm{C}$ with pulsatile perfusion, maintaining a minimum pressure of 70 $\mathrm{mm} \mathrm{Hg}$. The last patient had a combination of pulsatile and nonpulsatile perfusion. The median CPB and crossclamp times were 88 and 101 minutes, respectively, without circulatory arrest.

Fetal monitoring. Placental and fetal blood flows were monitored by means of transvaginal ultrasonography with an $8-\mathrm{MHz}$ probe. The maternal uterine arteries and fetal umbilical artery, descending aorta, and ductus venosus were identified with the aid of color Doppler ultrasonography, and frequency shift traces were obtained by means of pulsed-wave Doppler ultrasonography. In the first 3 patients the lowest fetal heart rate recorded was 70 beats/min, which recovered by the completion of operation. In patient 4 the fetal heart rate decreased to 40 beats/min. Before this gradual decrease, the mode of perfusion was changed from pulsatile to nonpulsatile, and an infusion of nitroglycerin was started in the hope of avoiding a further decrease in heart rate and placental vasoconstriction (Figure 1).

Outcomes of pregnancy. The first 3 patients underwent elective cesarean section at 38 weeks' gestation and were delivered of normal newborns. In patient 4 the scans performed at 1 and 4 days after the operation were completely satisfactory. However, the scan before discharge showed features of hydrops. This was repeated after discharge, and the fetus continued to show signs of hydrops. She underwent termination at 17 weeks' gestation.

\section{Discussion}

CPB during pregnancy is associated with a maternal mortality rate of $3 \%$ to $15 \%$ and a fetal mortality of $20 \%$ to $33 \% .{ }^{1}$ In the cases described, the maternal outcome was favorable in all, and the fetal outcome was favorable in 3 . One of the pregnancies was terminated, possibly as a result of the use of nonpulsatile perfusion. Because of the increase in cardiac output, red cell mass, and oxygen consumption during pregnancy, the standard approach to CPB (ie, hemodiluted, nonpulsatile, and low flow) might be detrimental. In our patients $\mathrm{CPB}$ was performed at $35^{\circ} \mathrm{C}$ to avoid fetal arrhythmia and uterine contractions. Fetal bradycardia is known to develop at the start of CPB and return to normal after its establishment. There are reports of return of fetal heart tunes after surgical intervention despite their complete disappearance during $\mathrm{CPB}$ not resulting in fetal mortality. ${ }^{2}$ 


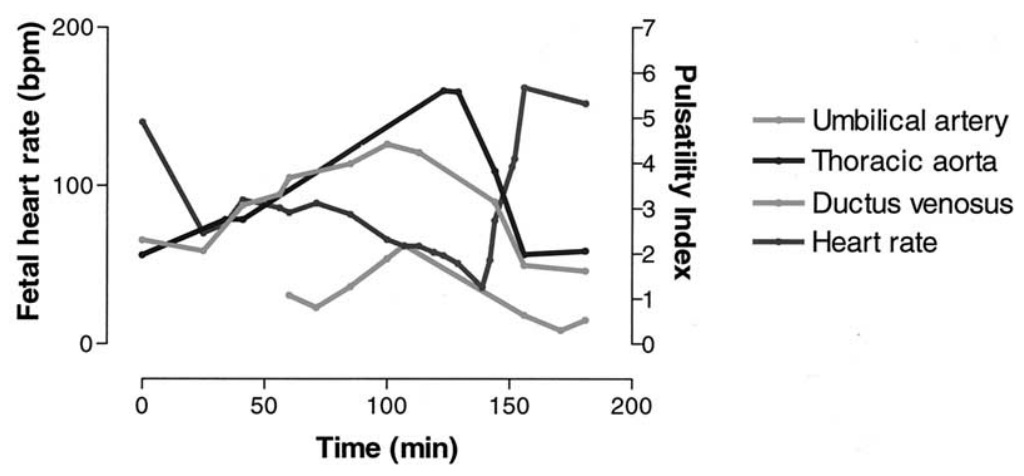

Figure 1. Change in fetal heart rate and pulsatility index (an index of resistance) in patient 4. CPB is established at 35 minutes and terminated at 145 minutes. Pulsatile perfusion was changed to nonpulsatile perfusion at 100 minutes.

Experimental evidence suggests that there is a prostaglandinmediated increase in placental vascular resistance that might cause a reduction in placental blood flow. ${ }^{3}$ Both indomethacin and steroid administered during fetal cardiac bypass have been shown to prevent the increase in placental vascular resistance. ${ }^{4}$ Pulsatile pressure is thought to reduce uterine contractions by releasing endothelium-derived growth factor from the vascular endothelium. Champsaur and colleagues ${ }^{5}$ have shown that pharmacologic blockade of endothelium-derived growth factor after 30 minutes of pulsatile flow returns fetal hemodynamics to continuous flow conditions, suggesting that nitric oxide might be released by fetal endothelium during pulsatile bypass.

With full maternal and fetal monitoring and attention to CPB, particularly the use of pulsatile perfusion, the risks to both the mother and the fetus can be minimized.

\section{References}

1. Chambers CE, Clark SL. Cardiac surgery during pregnancy. Clin Obstet Gynecol. 1994;37:316-23.

2. Mahli A, Izdes S, Coskun D. Cardiac operations during pregnancy: review of factors influencing fetal outcome. Ann Thorac Surg. 2000; 69:1622-6.

3. Hawkins JA, Clark SM, Shaddy RE, Gay WA. Fetal cardiac bypass: improved placental function with moderately high flow rates. Ann Thorac Surg. 1994;57:293-7.

4. Sabik JF, Heinemann MK, Assad RS, Hanley FL. High-dose steroids prevent placental dysfunction after fetal cardiac bypass. $J$ Thorac Cardiovasc Surg. 1994;107:116-25.

5. Champsaur G, Vedrinne C, Martinon S, Tronc F, Robin J, Ninet J, et al. Flow-induced release of endothelium-derived relaxing factor during pulsatile bypass: experimental study in the fetal lamb. J Thorac Cardiovasc Surg. 1997;114:738-45. 